\title{
El rol de la mujer militar y su aporte a la sociedad en el posacuerdo de paz ${ }^{1}$
}

https://doi.org/10.21830/9789585318304.10

\author{
Mónica Paola Ortega Guzmán ${ }^{2}$ \\ Juan Fernando Gil Osorio \\ Escuela Militar de Cadetes "General José María Córdova”
}

\section{Resumen}

El espacio de la mujer en las Fuerzas Militares implica una lucha permanente por los derechos y la igualdad, así como pensar un rol femenino militar dispuesto para los deberes propios de la institución en pos de un ejercicio constante de la dignidad y los derechos humanos. Esto se ha traducido en un actuar dentro y fuera de las Fuerzas Militares para acatar la misionalidad militar y asumir la defensa de los derechos a la igualdad y la paz. De esta forma, la mujer militar se ha convertido en un agente clave para la construcción de paz y el cumplimiento de los fines constitucionales de la sociedad colombiana en el posacuerdo.

Palabras clave: carrera militar; equidad; Fuerzas Militares; igualdad; mujer; paz

\section{Introducción}

Este capítulo busca comprender en qué medida la inclusión de las mujeres en las Fuerzas Militares (FF. MM.) y la implementación de una perspectiva de género en el quehacer militar puede reconfigurar una serie de roles — gestados en el marco de los derechos humanos (DD. HH.) - que contribuyan a una misionalidad para la implementación de los Acuerdos de Paz. Para ello, se analiza desde una perspectiva

1 Este capítulo presenta los resultados del proyecto de investigación "Observatorio de derecho operacional" del Grupo de Investigación en Ciencias Militares, de la Escuela Militar de Cadetes "General José María Córdova” (ESMIC), categorizado en B por Minciencias y con código de registro COL0082556. Los puntos de vista y los resultados de este artículo pertenecen a los autores y no reflejan necesariamente los de las instituciones participantes.

2 Abogada (Universidad Católica de Colombia). Posgrado en Derecho Constitucional y Administrativo.

3 Doctorando en Derecho (Universidad Externado de Colombia). Máster en Derechos Humanos y Democratización (Universidad Externado de Colombia y Universidad Carlos III de Madrid). Especialista en Derechos Humanos y Derecho Internacional Humanitario (Universidad Externado de Colombia). Abogado (Universidad de Medellín). Director del Observatorio de Derecho Operacional de la Escuela Militar de Cadetes “General José María Córdova”. Orcid: https://orcid.org/0000-0002-6605-6846 - Contacto: juan.gil@esmic.edu.co 
histórico-social la forma en que la mujer ganó su lugar en las FF. MM. y los matices que determinaron la construcción de su rol, que se establece a partir del enfoque de los derechos humanos. Este escenario se amplía con el análisis de las complejidades del posacuerdo colombiano, con el fin de plantear un marco inclusivo de acción militar que contribuya efectivamente a la construcción de la paz social desde el actuar de la mujer militar.

Esto permitirá que los análisis con perspectiva de género en las FF. MM. no se limiten a la inclusión dentro de la instrucción de la militar en formación, sino que comprenda estos procesos como una transformación en los roles femeninos propios de las militares en su ejercicio profesional.

Históricamente, toda reivindicación sobre la conquista de los derechos de las mujeres ha implicado una tensión social entre los imaginarios, sostenida por la idea patriarcal que establece un escenario de privilegios masculinos enfrentada a las exigencias por desarraigar aquellas concepciones que refuerzan la idea de la mujer como ciudadana de segunda mano. La figura de las FF. MM. como un conjunto de instituciones castrenses pertenecientes a la institucionalidad estatal permite pensar el proceso de la inclusión de las mujeres a sus filas como una dinámica que, además de permitir un avance en la garantía de los derechos de las mujeres, establece un escenario de legitimidad institucional mayor al ampliar la diversidad en la misionalidad militar, lo que contribuye al avance de los procesos humanizadores de los ejércitos.

Es entonces cuando evoluciona el análisis de la mujer en las FF. MM. más allá de sus condiciones como miembros en instrucción o en favor de su cuota participativa en altos mandos militares. Esta investigación busca contribuir no solo en la caracterización del imaginario alrededor del rol de la mujer militar (a partir de una observación de la historia de la garantía de sus derechos y sus avances en la influencia dentro de la institución), sino en la posibilidad de establecer mecanismos que contribuyan a la equidad en el desarrollo de sus labores dentro del proceso de posacuerdo de paz.

Las sociedades mantienen estructuras de desigualdad en las que los hombres fungen como actores principales en todos los escenarios y los conflictos, lo cual limita la participación de las mujeres en la resolución de confrontaciones y las reduce a simples observadoras o víctimas (Beauvoir, 1949). Sin embargo, la falta de garantías igualitarias para la participación no ha alejado a las mujeres de los conflictos, ya que esto no las priva de desempeñar otros roles aparte de aquellos relacionados con la familia, como el ser cuidadoras, sino que les permite llegar a ser personalidades políticas, activistas por la paz o incluso combatientes (Vargas \& Díaz Pérez, 2018). 
Es necesario, entonces, comprender que aun cuando no se goce de un escenario de igualdad material entre sexos, debe propenderse, en cada uno de los espacios de construcción social, a establecer condiciones para la conciencia de la desigualdad y espacio para los actos restaurativos y para el saneamiento de las brechas en la búsqueda de una sociedad sin desigualdades.

Organismos internacionales han evidenciado que las mujeres en los procesos de construcción de paz ayudan a fortalecer el tejido social y facilitan que en ese ejercicio se reconozcan violencias invisibilizadas, lo que permite aumentar la inclusión y la diversidad. En efecto, su rol de mediadoras responde a los intereses de un mayor número de partes involucradas y da lugar a la participación necesaria para una paz más sostenible (Naciones Unidas, 2014). Este reconocimiento internacional de la importancia de la mujer en la consolidación de paz permite una nueva lectura de los derechos humanos con perspectiva de género aplicable a los procesos de paz.

En este sentido, la inclusión de la mujer en escenarios de resolución de conflictos requiere su comprensión por fuera de los típicos roles determinados (que las conciben de manera unidimensional) e implica su inclusión en conceptos internacionales que resaltan que ellas no solo son víctimas, sino que también se encuentran mujeres combatientes, diplomáticas y agentes del Estado:

Dicha inclusión se hace a partir de la identificación de los roles individuales dentro del conflicto de hombres y mujeres, donde si bien el rol de los hombres se extiende de manera generalizada sobre la actuación violenta en combates y enfrentamientos, las mujeres cumplen una función doble dentro del conflicto. (Calderón et al., 2017)

Esta investigación, en consecuencia, buscó rescatar la labor de la mujer militar como una conquista participativa y como facilitadora en los procesos de armonización en distintas misiones de las Fuerzas Militares con relación a la implementación de los Acuerdos de Paz.

\section{El rol de la mujer en las FF. MM. colombianas}

A lo largo de la historia, la mujer siempre ha tenido un papel secundario en los distintos aspectos en los que se desarrolla en la sociedad. El rol dentro de las Fuerzas Armadas no ha sido la excepción, por lo que los espacios que estas han ganado dentro de las Fuerzas Militares se han determinado a través de la garantía y protección de los derechos humanos, más específicamente, el de la igualdad. Este mismo le brinda a las mujeres la oportunidad de sumar sus habilidades en todos los escenarios necesarios para negociar y obtener la paz (Camacho, 2014). Así pues, este fenómeno de asimetría se explica en el entendido de que 
En América Latina [...] la discusión sobre la participación de mujeres en las Fuerzas Armadas refleja su realidad más general, centrada en la invisibilidad del trabajo femenino y sus dificultades de incorporación igualitaria al mercado laboral. Esto es particularmente cierto para la mayoría de los países del Caribe. (Bobea, 2008)

Esta inclusión pretende que las mujeres, por medio de su participación, diseñen estrategias de prevención y solución de conflictos, todo esto tomando en cuenta los lineamientos internacionales en materia de equidad e inclusión de género, lo que genera mayor participación de ella en las Fuerzas Armadas colombianas. Esto hace a un lado la idea patriarcal de los hombres como único actor militar, pero para que esta participación se vea materializada es necesario que los avances en lo relacionado con los roles de la mujer en la vida militar vayan más allá de las acciones meramente organizativas y se les haga partícipes de los altos mandos militares según sus destrezas y aptitudes.

\section{Desarrollo histórico de la mujer militar}

En términos históricos, se estima que la primera mujer en hacer parte de la Fuerza Militar en el territorio nacional fue la prócer Policarpa Salavarrieta, toda vez que formó parte del Ejército Patriota de los Llanos en 1810. Se cuenta con registros que señalan que la siguiente mujer con rol militar fue Clara Elisa Narváez Arteaga, en 1932, quien se convirtió en la primera soldado al participar en la guerra entre Colombia y Perú, en función activa y de socorro a los heridos en el combate. No obstante, la primera participación con un marco jurídico de respaldo fue en 1976, mediante el Decreto 2129, que permitió el ingreso de mujeres al Ejército en la categoría de oficiales del cuerpo administrativo en áreas de medicina, derecho, administración y educación (Hussain-Talero, 2018, p. 106).

Hasta 1979 se logró la primera incorporación de mujeres en el cuerpo administrativo de la Fuerza Aérea. Esta y la década siguiente fueron importantes para la mujer militar, ya que, además de lo mencionado, en 1983 el Ejército permitió la vinculación suboficiales en sanidad, contabilidad, archivo y educación, y en 1984 ingresaron las primeras mujeres profesionales a la Armada Nacional, y ocho años más tarde esta última admitió el ingreso de mujeres como suboficiales (HussainTalero, 2018, p. 106).

En 2009, se nombró la primera brigadier general de la Policía Nacional, lo que implicó un claro avance de las mujeres militares en los roles de mando, ya que la labor de la mujer militar llevaba desde la época de los setenta relegada al ámbito 
administrativo (De la Hoz Flórez, 2018, p. 19). Esto determinó un rol femenino en las Fuerzas Militares al margen de la labor en misión, determinó un nicho de coordinación y organización dentro de la institución en el que se afianzó la mujer militar. Además, en los procesos de restructuración a partir del Plan Damasco, el Ejército integró a las mujeres al conteo de sus reservas, con lo cual amplió el espectro de desarrollo del rol de la mujer militar (De la Hoz Flórez, 2018, p. 19).

Es de resaltar que, gracias a la Organización del Tratado del Atlántico Norte (OTAN) y el establecimiento de una oficina de incorporación de mujeres en sus fuerzas en 1998, se priorizó en el Estado colombiano la discusión para integrar de manera efectiva a las mujeres militares en la diversidad de funciones propias de las Fuerzas Militares. La promoción de la equidad de género logró una vinculación material de las mujeres en las fuerzas de paz, en obediencia al escalamiento de estas discusiones en la ONU. Esto permite que se comprenda en términos generales el rol de la mujer en la construcción del posconflicto como un activo, ya que gracias a la narrativa de historias en las que prevalecen las gestiones de las lideresas políticas para la materialización de los derechos, la mujer adquiere la posibilidad de ser un referente para su comunidad. Así, pues, gracias a la Resolución 1325 del Consejo de Seguridad de la ONU esta visión que puede comprenderse como

una reflexión sobre el papel de la mujer como mediadora y negociadora en la resolución de conflictos, no sólo por ser un derecho reconocido en la normativa internacional, sino porque, además, sus valores, su opinión y sus acciones son imprescindibles para las soluciones adoptadas. Lo son porque suponen prácticamente la mitad de la población en los actuales teatros de operaciones (especialmente Afganistán), porque tradicionalmente, por su maternidad o por su proceso de socialización, han priorizado los valores de diálogo, de solución de conflictos y de anteponer la seguridad humana y evitar la violencia. (Gallardo Rodríguez, 2012)

Las FF. MM. colombianas han implementado medidas acordes a los lineamientos internacionales en materia de equidad e inclusión de género, gracias a lo cual se ha fomentado la participación de las mujeres en la defensa de la soberanía nacional $y$, consecuentemente, en el sostenimiento del orden constitucional para la construcción de la paz. La participación cada vez mayor de las mujeres en las Fuerzas Militares de Colombia es un reflejo de cambios estructurales globales en los que ellas ocupan un número significativo en las filas y mandos, en contraste con su integración a las dinámicas militares que se hizo global a mediados de la década de los ochenta. 


\section{Caracterización del rol femenino en las FF.MM.}

El avance del rol de la mujer militar no es un asunto que pueda medirse con solo cifras de participación, sino que debe pensarse concibiendo el hecho de que la estructura militar ha sido pensada desde un enfoque androcentrista y que la materialización efectiva de equidad para los y las militares se evidenciará solo en la medida en que se vayan reduciendo los conceptos que comprendan a los hombres como únicos actores del contexto militar. La Resolución 1325 de 2000 de la ONU evidenció la existencia de modelos en los que se replicaban las estructuras patriarcales en las fuerzas militares, reforzadas por algunas prácticas propias del ámbito castrense (Cely Calderón, 2019).

Para ello es primordial observar que, aun cuando los avances en la acción de la mujer militar han sido significativos, muchos de ellos siguen bajo el estigma de ser el único espacio de despliegue para una mujer. Su actuar, en efecto, sigue siendo transversalizado en Colombia por las mencionadas visiones patriarcales, cuando las FF. MM. deben adecuarse a los valores constitucionales que hacen parte del núcleo esencial de defensa que determina la misionalidad militar, de forma que sea posible un avance hacia los fines del Estado:

La Nación tendrá para su defensa unas Fuerzas Militares permanentes constituidas por el Ejército, la Armada y la Fuerza Aérea. Las Fuerzas Militares tendrán como finalidad primordial la defensa de la soberanía, la independencia, la integridad del territorio nacional y del orden constitucional. La ley determinará el sistema de reemplazos en las Fuerzas Militares, así como los ascensos, derechos y obligaciones de sus miembros y el régimen especial de carrera, prestacional y disciplinario, que les es propio. [...] Son fines esenciales del Estado: servir a la comunidad, promover la prosperidad general y garantizar la efectividad de los principios, derechos y deberes consagrados en la Constitución; facilitar la participación de todos en las decisiones que los afectan y en la vida económica, política, administrativa y cultural de la Nación; defender la independencia nacional, mantener la integridad territorial y asegurar la convivencia pacífica y la vigencia de un orden justo. Las autoridades de la República están instituidas para proteger a todas las personas residentes en Colombia, en su vida, honra, bienes, creencias, y demás derechos y libertades, y para asegurar el cumplimiento de los deberes sociales del Estado y de los particulares. (Constitución Política de Colombia, 1991, arts. 2 y 217)

En este orden de ideas, el aporte de las Fuerzas Militares al escenario del posacuerdo debe ser sustancial, toda vez que su función no se reduce a la confrontación armada, tal como lo señala la constitución colombiana.

Es aquí donde las mujeres militares juegan un papel destacado como figura articuladora para la construcción de paz, ya que su historia dentro de la institución 
está atravesada por una serie de acciones para el reconocimiento de sus derechos y de sus potencialidades humanas. Gracias a esto, se hace posible la creación de un escenario de cambio y diálogo, es decir, el reconocimiento de sus derechos se posibilitó a partir de roles de negociación y viabilidad de diálogos en DD. HH. (Comando General de las Fuerzas Militares de Colombia, 2017, pág. 49). Esto implica que el rol femenino militar funcione con menor dificultad en tareas de cohesión social. Como se ha señalado, se ha creado desde una función trasmutadora de sus contextos, que cuenta con un apoyo internacional por representar un ejercicio constante de la garantía de los derechos humanos.

\section{El liderazgo de la mujer militar}

El liderazgo del rol militar femenino rompe con la estructura clásica del establecimiento de las funciones desde las órdenes para implementar una comunicación desde un ejercicio de motivación y educación. Esto contribuye a la creación de un entorno con mayor estabilidad emocional, que permite un ambiente más sano para la toma efectiva de decisiones y la implementación de estrategias colaborativas (Hussain-Talero, 2018, p. 96)

El proceso de integración del concepto del enfoque de género en las Fuerzas Militares ha permitido un avance en el cumplimiento de las disposiciones de la Resolución de la ONU 1325 de 2000 y en el rol que las instituciones militares desempeñan en las respectivas sociedades. Es por ello que la ONU, en su visión de los procesos de paz, enfatizó en la necesidad de aumentar el número de mujeres en las fuerzas y misiones militares.

En 2015, la Fuerza contaba con una totalidad del 6\% de uniformadas pertenecientes a los efectivos movilizados en las distintas operaciones internacionales de los países miembros de la OTAN y un 6,4\% en las operaciones comandadas por la Alianza misma, sin embargo, en relación con las operaciones de la ONU, las mujeres representan actualmente entre un 0 y un $4 \%$ de los contingentes. Esto permite comprender que, pese a la existencia de la Resolución no hay una participación efectiva y suficiente de la mujer militar para que su rol sea significativo en el sostenimiento de los procesos de paz, por lo tanto, la inclusión debe permitir ampliar este escenario.

La participación de mujeres en misiones internacionales de paz, negociaciones de paz y procesos de desarme aumenta claramente la eficacia de estos, dado que se establecen criterios de veeduría y denuncia, y se prioriza que toda disposición se dé 
en concordancia con un mínimo que posibilite el goce de los derechos humanos, tal como se ha evidenciado en los procesos de la OTAN:

En diciembre de 2007, la OTAN y sus socios acordaron poner en práctica la Resolución 1325 y ampliar el papel de la mujer en las operaciones de las Naciones Unidas y especialmente entre los observadores militares y la Policía civil. Compromiso que quedó plasmado en el informe CWINF Guidance for NATO Gender Mainstreaming. Este documento significó la primera contribución de la Alianza al desarrollo de un enfoque general de la perspectiva de género en sus operaciones militares. Se inicia a partir de este momento un nuevo papel para la mujer militar perteneciente a los países aliados en los escenarios de conflicto, que puede, además de sus tareas fundamentales, ser una comunicadora especial y persona influyente sobre el sector femenino de la población de la nación anfitriona, especialmente en las culturas musulmanas. Papel que por primera vez desde el ingreso de la a mujer a las FAS es competencia de las soldados por razón de su género. (Gallardo, 2012, p. 147)

Con tal finalidad, aparece entonces la Resolución 2242, que facilita la capacitación de mujeres para participar en estos procesos y atiende a la formación y el análisis del impacto de la presencia de las mujeres y las estrategias para su inclusión efectiva.

Esta Resolución permite asumir como compromiso institucional la integración efectiva de la perspectiva de género en el análisis del conflicto. A partir de esto se hace un llamado a los Estados para que dupliquen, a lo largo de los próximos cinco años y con los recursos existentes, el número de mujeres en los contingentes militares y en la policía que trabaja en las operaciones de mantenimiento de la paz de la ONU.

\section{El deber de la inclusión de las mujeres en el mundo militar}

La inclusión de las mujeres en la carrera militar en Colombia se remonta a 1976, cuando mediante el Decreto 2129 se establece el ingreso al escalafón militar en la categoría de Oficiales del Cuerpo Administrativo. Con ello se creó la posibilidad de que doce mujeres profesionales ingresaran a las filas armadas para prestar sus servicios como parte de las Fuerzas Militares (Congreso de la República, 1976). Este antecedente amplió los escenarios para que las mujeres empezaran a escalar posiciones en la lucha por lograr la igualdad y la equidad entre géneros en la carrera militar. Ahora bien, el concepto de igualdad también fue reconocido 
como un derecho fundamental un par de décadas más tarde, en el artículo 43 de la Constitución Política:

ARTíCUlO 43. La mujer y el hombre tienen iguales derechos y oportunidades. La mujer no podrá ser sometida a ninguna clase de discriminación. (Constitución Política de Colombia, 1991)

Tal vocación para la igualdad permite comprender que esta se aplica en todos los roles de la vida, con la finalidad de que la mujer tenga las mismas posibilidades de demostrar sus habilidades en las distintas formas de desarrollo personal y profesional. Así se crea la necesidad del Estado de propender a la materialización de la igualdad de oportunidades, dado que la mujer goza de los derechos consagrados en la carta de derechos fundamentales, que se alinean con la Convención Interamericana sobre Violencia contra la Mujer, que fue aprobada en 1996 mediante la Sentencia C-408/96 de la Corte Constitucional, que consideró que este tipo de protección reforzada para los derechos de la mujer nunca está de más, dado que

desafortunadamente en la práctica la violencia y la discriminación contra la mujer se encuentran muy extendidas, pues son un ejercicio de poder que deriva en gran medida de las relaciones inequitativas que subsisten entre mujeres y hombres. (Sentencia C-408/96)

En este mismo sentido, existen factores que generan desigualdad y limitan las posibilidades de que las mujeres militares avancen en su carrera, por lo que era necesario un cambio en la organización interna de las instituciones de las FF. MM. Así, en el año 2000, surge la Ley 581 de 2000, que decreta la activa participación de las mujeres en los cargos de dirección de las entidades del Estado y en las ramas del poder público. Incluso en el evento de que la designación se hiciera por ternas, estas siempre deberán tener mujeres como candidatas, y en caso de elección por listas, estas serán equitativas en la cantidad de mujeres y hombres. Estas determinaciones tienen por objeto garantizar la efectiva integración de la mujer en las decisiones importantes del país, de forma que pongan en práctica sus capacidades y habilidades en campos como la concertación y la organización, factores determinantes para la adecuada administración de entidades en los órdenes nacional, departamental, distrital y municipal (Congreso de la República, 2000).

De la misma forma, mediante la doctrina Damasco, promulgada en virtud de la Disposición 004 del 26 de febrero de 2016, se pretende que las mujeres también hagan parte de las reservas del Ejército y constituyan un componente integral y fundamental de las Fuerzas Armadas (Congreso de la República, 2016). En 
la carrera militar, más específicamente, se han generado decisiones mediante las cuales se propende a la inclusión y la igualdad para la mujer, como la sentencia C-659/2016 de la Corte Constitucional, mediante la cual se precisa que la mujer tiene la posibilidad de entrar como pie de fuerza activo en operaciones de combate. Con ello se busca que en el desarrollo de la actividad militar las personas sean designadas en favor de la necesidad y de sus habilidades para desarrollar dichas tareas indistintamente del género. Esto también se aplica a la prestación del servicio militar, voluntario u obligatorio, tomando en cuenta el artículo 4 de la Ley 1861 de 2017, que establece que las mujeres también pueden prestar el servicio militar de forma voluntaria cuando así lo deseen (Sentencia C-408/96).

\section{Los derechos humanos para las garantías de la mujer militar}

A pesar de que los derechos y libertades fundamentales se encuentran garantizados para todas las personas y se consideran intrínsecos e inalienables, a lo largo de la historia se ha generado normatividad de todo tipo a nivel nacional e internacional para establecer cómo se deben garantizar y que conductas se consideran violaciones directas de estos. Para las mujeres, la lucha por este reconocimiento ha sido más larga y difícil, por el hecho de que, dentro de las estructuras patriarcales dominantes, se le considera un ser inferior:

La división de las cosas y de las actividades (sexuales o no) de acuerdo con la oposición entre lo masculino y lo femenino recibe su necesidad objetiva y subjetiva de su inserción en un sistema de oposiciones homólogas, alto/bajo, arriba/abajo, delante/detrás, derecha/izquierda, recto/curvo (oblicuo) (y pérfido), seco/húmedo, duro/blando, sazonado/soso, claro/oscuro, fuera (público)/dentro (privado), etc. (Bordieu, 2000, p. 9)

Es por esto que los Estados, a través de sus gobernantes, entidades públicas, organizaciones defensoras de derechos humanos y activistas, han luchado de forma incansable para garantizar una protección reforzada de estos derechos en la búsqueda de la equidad de género, lo que los ha llevado a unir esfuerzos de forma internacional, para reglamentar y aprobar tratados y leyes como los que se mencionaran en este acápite.

En 1994, la ONU estableció garantías para los derechos humanos y libertades de las mujeres, haciendo hincapié en el derecho a la igualdad y sus diferentes aspectos, tales como protección ante la ley, igualdad de acceso a las funciones 
públicas en su país y la participación en los asuntos públicos, incluyendo la toma de decisiones. Esta protección se fundamenta en garantizar el acceso de la mujer a cargos directivos y de decisión, en los que se tenga en cuenta su forma de pensar y su criterio, así como sus aportes a la solución de conflictos. Se reconoce también a la mujer como tejedora de la paz, debido a que siempre se ha enfrentado a situaciones de discriminación, por lo que su punto de vista viene desde la vivencia de las situaciones que afectan a la sociedad (Bobea, 2008) .

Es por esto que se consagra el derecho a un trato igualitario para la mujer $y$, en consecuencia, a vivir libre de discriminación alguna, a ser valorada y educada libre de patrones estereotipados de comportamiento y de prácticas sociales y culturales basadas en conceptos de inferioridad y subordinación. De esta forma, se le brinda a la mujer el valor correspondiente y la posición que le pertenece dentro de la sociedad como persona titular de derechos, tal y como se estableció en la Convención de Belém do Pará (Organización de las Naciones Unidas, 1994).

Por otra parte, la adopción de decisiones para hacerle frente al conflicto armado $^{4}$ es una responsabilidad esencial para el logro de la convivencia pacífica y del respeto por los derechos fundamentales. Por esto, la participación igualitaria de la mujer en la adopción de estas decisiones es una exigencia básica de justicia y democracia, necesaria para que se tengan en cuenta sus intereses porque sin la cooperación activa de la mujer no se podría conseguir el tan anhelado objetivo de igualdad, desarrollo y paz. Prescindir del punto de vista de este grupo de la población sería inequitativo e ineficaz para el anhelado objeto de la paz en Colombia (Organización de las Naciones Unidas, 1995a, p. 101).

En este orden de ideas, la ONU es reiterativa en subrayar la importancia de la participación de la mujer en pie de igualdad, así como en reclamar su intervención en todas las iniciativas encaminadas al mantenimiento y fomento de la paz y seguridad, objetivo que solo se cumplirá cuando se dé el aumento de su participación en los procesos de toma de decisiones en materia de prevención y solución de conflictos (Organización de las Naciones Unidas - Consejo de Seguridad, 2000).

4 Se comprende el conflicto armado, a la luz del DIH, como el enfrentamiento violento entre dos grupos humanos en una sociedad, cuyo resultado es masivo y, generalmente, ocasiona muertes y destrucción material (Calderón et al., 2017). 


\section{La labor humanitaria y las FF. MM.}

Cuando se genera un levantamiento armado, lo primero que se debe tener en cuenta es cuáles fueron las razones que lo originaron y cuáles las necesidades insatisfechas del sector de la población que se está levantando, ya sea por política internacional o por una conmoción interna. De esta forma, se diseña una estrategia consecuente, por medio de la cual el Estado pueda contrarrestar este ataque y retornar el orden (Valencia Tovar, 1994). Se debe tomar en cuenta, no obstante, que la respuesta a este levantamiento armado siempre debe estar regulado por los DD. HH. y los lineamientos establecidos por los tratados y las leyes nacionales e internacionales. Dichos lineamientos imponen el respeto y la garantía de los derechos humanos, es decir, humanizan el conflicto armado, ya que en estos conflictos los derechos fundamentales se ven gravemente afectados por la politización y el abuso de poder: en ocasiones, se toman decisiones que no son justas para la población más vulnerable (Organización de las Naciones Unidas, 1995a).

Cuando así sucede, se genera el desprestigio de los gobiernos, que trae como consecuencia la falta de apoyo de la comunidad internacional por la falta de garantías y las graves violaciones a la dignidad humana y las libertades de población civil. En consecuencia, los miembros de la fuerza pública deben actuar dentro de un sistema político democrático salvaguardando la dignidad humana y obrando dentro de un orden jurídico basado en la carta constitucional como garante del derecho internacional humanitario (en adelante, DIH) y de las normas civilizadas de la guerra. Solo así, puede el Estado ganar la confianza y credibilidad de la población que defiende y hacerla su aliado (Organización de las Naciones Unidas - Consejo de Seguridad, 2000).

\section{Referentes de la mujer militar en la labor humanitaria}

En la labor humanitaria se enmarcan aquellas acciones encaminadas a brindar ayuda a las víctimas del conflicto armado. Esto implica que los gobiernos y sus fuerzas armadas están en el deber de tomar las medidas necesarias para hacer frente a las emergencias humanitarias que se suscitan. Así está establecido desde 1864, cuando se firmó el primer Convenio de Ginebra, de acuerdo con el cual, las fuerzas armadas deben integrar en sus operaciones las normas humanitarias necesarias para sensibilizar y fortalecer sus operaciones sin incurrir en errores o infracciones de los derechos humanos. 
La asistencia humanitaria se rige jurídicamente en Colombia por el DIH, de acuerdo con los Convenios de Ginebra y las diversas resoluciones adoptadas por el Comité Internacional de la Cruz Roja (CICR). El pilar fundamental de estas normativas es la prohibición de los atentados contra el derecho a la vida, ya sea por acción u omisión, así como la defensa del derecho a un trato humano y digno, con el fin de preservar la vida y las necesidades básicas de las víctimas (Sentencia C-438/13).

Con relación al derecho internacional humanitario, se ha pronunciado la Corte Constitucional de Colombia en la Sentencia C-225/95, en la cual se establece que en el país el derecho internacional humanitario es válido en todo tiempo y que, además, opera de forma automática, lo que le genera un estatus de carácter imperativo:

Al tenor del artículo 53 de la Convención de Viena de 1969 sobre el derecho de los tratados, se entiende por norma ius cogens o norma imperativa de derecho internacional general "una norma aceptada y reconocida por la comunidad internacional de Estados en su conjunto como norma que no admite acuerdo en contrario y que solo puede ser modificada por una norma ulterior de derecho internacional general que tenga el mismo carácter". (Sentencia C-225/95)

De lo anterior se deduce que toda norma que resulte contraria a esto será nula, por lo que estas normas son de obligatorio cumplimiento para todos los Estados, incluso para los que no han aprobado los respectivos tratados con la finalidad de salvaguardar la vida y la integridad de la población civil, que resulta victimizada en estos conflictos.

Con el pasar del tiempo, la mujer ha escalado posiciones dentro de las FF. MM., actuando y haciéndose cada vez más visible por su trabajo y contribución en la institución, así como en la colaboración en la atención del conflicto armado, tal y como lo expresa la brigadier general Galvis, del Ejército Nacional:

[Ella] considera que ante un escenario en el que ponga en práctica un Acuerdo de Paz, la mujer militar tendrá la posibilidad de visibilizarse mucho más. Piensa que, aunque claramente ya son vistas con equidad dentro de la fuerza, en este nuevo escenario se abrirán nuevas puertas para que las 1.758 mujeres —hablando solo del Ejército- puedan entrar a competir de manera más equitativa con sus pares masculinos, de acuerdo con sus capacidades y los perfiles que necesita la institución. (Londoño, 2017) 


\section{El posacuerdo: la contribución de las mujeres militares a la construcción de la paz}

La relación circunstancial entre mujer y conflicto armado se da en un escenario en el que predominantemente las mujeres han sido sujetos pasivos y blancos de ataque, mientras que la carga político-cultural de los hombres ha sido la de ser perpetuadores y sujetos activos en el fuego cruzado (Calderón et al., 2017). La guerra, como escenario general, afirma una serie de características que históricamente se han sostenido sobre la imagen de virilidad, se piensa y se desarrolla desde la defensa y el ataque de los hombres en el frente de batalla, mientras que las mujeres son sujetos accidentales o bajas en las dinámicas bélicas, por lo tanto, la mujer se asume comúnmente como el sujeto salvado por la heroica figura masculina militar o en virtud de los roles típicamente impuestos por su feminidad (Huertas Díaz et al., 2017).

Es necesario aclarar que el conflicto armado colombiano se ha servido de instrumentalizar a la mujer como arma y territorio de disputa de guerra, relegándola a la condición de víctima de violencias sexuales y otros hechos crueles y degradantes. Por una parte, las mujeres dentro de las FF.MM. están en una constante lucha por la ganancia de participación y de derechos, acogidas a la misionalidad militar y, por otra, son víctimas del conflicto:

El conflicto armado ha llevado a las mujeres a asumir nuevos roles, dejando su papel de víctima a un lado y pasando a tener una participación más activa en el conflicto, ya sea como miembro activo de enfrentamiento tanto en las filas de las organizaciones al margen de la ley, como combatiente y en la esfera política, por una parte. Por otra parte, a pesar de que las mujeres han sido integrantes activas de procesos de reclamaciones y luchas sociales, en el curso del conflicto se empiezan a hacer más visibles y se transforman en voceras o promotoras de paz desde sus ámbitos sociales más cercanos, sea su hogar, su barrio o comunidad. A su vez, la mujer se ha empoderado reconociéndose como sujeto de derechos, por lo que empieza a ejercer actividades de cabildeo y a trabajar en espacios antes desconocidos como lo es la política en pro de la justicia social y de la construcción de paz. (Barros \& Rojas, 2015)

Esto supone un reto mayor en la inclusión de mujeres militares en los procesos de paz, toda vez que tienen un papel crucial para establecer un escenario en el que se implemente una perspectiva de género a la hora de concebir a las mujeres en el marco del conflicto. Del mismo modo es necesario reconocer a la mujer como sujeto activo en el conflicto, así como reivindicar su presencia activa como mujer militar y la manera en que ha asumido labores de mando para reconfigurar su rol (Ramírez, 2016). 
De hecho, el contexto de conflicto armado colombiano contó con una mujer militar importante para los acuerdos, la teniente de navío Juanita Millán Hernández, que se integró a los procesos del Acuerdo de Paz de 2016 por medio de la mesa de negociación. La teniente describe su experiencia como profesional formada en las Fuerzas Militares como un análisis dialógico con el conflicto:

Al inicio de mi carrera militar, mi idea de terminar ese conflicto y lograr la paz no era precisamente a través de un proceso de paz, pero la vida, el conocer más la realidad de nuestro país y mis creencias me fueron haciendo cambiar de estrategia para ayudar desde mis posibilidades a acabar con el conflicto, así que me preparé por lo menos desde el punto de vista académico, para aportarle a la paz. (Millán, 2017)

La teniente ocupó en su trabajo los roles administrativos que han sido asignados a las mujeres militares a lo largo de la historia reciente. Esto fue lo que configuró en ella una comprensión del mando militar a partir de mecanismos de comunicación y negociación, lo que facilitó su visión del proceso en el marco del diálogo de paz. Ella es, sin duda alguna, un referente importante en la comprensión de la versatilidad del rol de mujer militar en épocas de posacuerdo, pues conoce de primera mano las narrativas tanto del proceso como de la firma del acuerdo y su implementación:

Sé que, como nación, nos queda un camino muy largo que recorrer, sé que tenemos retos mayores, pero tengo la confianza en que tanto los hombres y mujeres de las Farc que están caminando en estos momentos hacia un nuevo futuro, como nosotros desde las Fuerzas Militares y la sociedad civil encontraremos un objetivo común. A la par de esos pasos de las Farc, hay cientos de personas haciendo que esto pase, planeando la logística, coordinando la seguridad, planeando los desplazamientos, haciendo magia en los territorios para que esa caminata sea la última que tengan que hacer. (Millán, 2017)

\section{Las acciones militares más allá de la función armada, contexto del posacuerdo colombiano}

La comprensión de las FF. MM. como una institución de defensa de la soberanía, la honra y la dignidad del pueblo colombiano es el punto de partida para observar las múltiples acciones de las uniformadas en virtud de un sinnúmero de deberes. La protección del territorio, la atención a situaciones de riesgo natural y el acompañamiento de labores gubernamentales son algunas de las acciones distintas 
a las bélicas realizadas por ellas. No debe olvidarse que las Fuerzas Militares cuentan con un amplio contexto jurídico nacional e internacional para cada una de las misionalidades y que cada etapa de instrucción y aprendizaje cuenta con una amplia cobertura de enseñanza en materia de DD. HH.

Históricamente la interacción ente civiles y FF. MM. se fundamenta en la protección y la seguridad, así como los mecanismos misionales y la logística necesarios para el establecimiento de una soberanía y protección territorial, sin embargo, los ejércitos han interactuado con organizaciones no militares en virtud del DIH y del derecho internacional de los derechos humanos (en adelante, DIDH), como el Comité Internacional de la Cruz Roja y Médicos sin Fronteras.

Las emergencias naturales son uno de los escenarios sociales en los que las Fuerzas Militares han intervenido más allá de la acción de combate. Existe en este escenario un apoyo importante proporcionado por el sector defensa cuando se notifican casos de emergencias domésticas. En efecto, con la creación (mediante la Ley 1523 de 2012) del Sistema Nacional de Gestión del Riesgo de Desastres, el Estado colombiano establece una ruta de acción para conocer y reducir las calamidades ocasionadas por desastres naturales a partir de la coordinación entre las instituciones nacionales y las entidades territoriales, entre ellas, las FF. MM. Este sistema involucra de manera general a todas las entidades públicas para el desarrollo de políticas económicas, sociales y ambientales (Donadio et al., s. f.)

El derecho internacional, a partir del Estatuto de Roma, ha limitado la participación e interacción de las fuerzas armadas en acciones directas con civiles, sin embargo, el contexto de las interacciones humanitarias permite un rol mayor al sector de la defensa y la seguridad, como garante de la integridad de las ONG y de terceros en la labor humanitaria. Gracias a estos escenarios se desarrolla la llamada cooperación cívico-militar (CIMIC) que se traduce en los lineamientos necesarios para las relaciones de este tipo en el marco de la labor humanitaria y el DIDH (Instituto de Estudios sobre Conflictos y Acción Humanitaria, 2010).

En Colombia, en 2016, se dio un antecedente importante en este escenario: una miembro activa de las Fuerzas Militares, la teniente Jéssica Alejandra Molina Figueroa, asumió la misión de limpiar las minas antipersona en todo el territorio colombiano. Esta misión humanitaria se dio en el marco de un escenario de acuerdo político de paz entre el gobierno y las FARC, uno de los grupos armados organizados más determinantes en el conflicto armado interno colombiano.

La teniente explicaba la tensión que implicaba el desminado por el riesgo de explosión y el cargamento pesado que tanto ella como sus subalternos debían cargar. Pese a que esta labor ponía en riesgo la vida de los miembros de las FF. MM., 
tuvo un menor reconocimiento social y mediático que el que tuvieron los uniformados destinados a la confrontación armada directa (Calderón, 2019).

Hay que señalar que, en información proporcionada por Descontamina Colombia al diario Vanguardia, de enero de 1985 a septiembre de 2019 se han registrado cerca de 11.769 víctimas por minas antipersona - lo suficiente como para poblar un municipio del tamaño de Curití (Santander) - y se ha cobrado la vida de 2.296 personas a lo largo de esos años. Entre las víctimas mortales se encuentran militares, policías, campesinos, niños y niñas (Calderón, 2019). La labor de la teniente le da a las Fuerzas Militares dos enfoques importantes: uno relacionado con la labor militar fuera de lo armamentístico y otro que muestra una contribución sustancial de la mujer en las funciones de las FF. MM.

\section{Las mujeres en la construcción de paz y su rol transformador}

Al hablar de la mujer dentro del conflicto armado, esta se relaciona en primer lugar como víctima, por estar dentro del grupo expuesto a mayor riesgo de violaciones de DD. HH. y derechos fundamentales. En consecuencia, los organismos internacionales ofrecen garantías y protección especial (Cely Calderón, 2019), por ejemplo, en el 2000, el Consejo de Seguridad de las Naciones Unidas dictó la Resolución 1325, en la que expresó su preocupación por el número de mujeres que conformaba la población civil perjudicada por los conflictos armados. En contraste, reconoció el importante papel por ellas desempeñado en la prevención y solución de los conflictos y en la consolidación de la paz (Organización de las Naciones Unidas, 2000).

Es así que día a día el papel desarrollado por la mujer en el posconflicto cobra mayor relevancia por sus incansables aportes para el logro de la paz. Por ello, la labor de la mujer militar se considera de capital importancia en la mediación y decisión frente a las consecuencias de la guerra que afectan a la sociedad civil. Su voz es escuchada por ser parte de la solución y no solo por ser víctimas mayoritarias de los actos de violencia.

Muchos de estos avances de la participación de la mujer, así como las normativas que los sostienen, son movidos por el contexto bélico de naciones como la república de Ruanda y la antigua Yugoslavia, donde las mujeres fueron las principales víctimas, por lo cual se adoptó una perspectiva de género dentro de las iniciativas internacionales de paz (De la Hoz Flórez, 2018, pp. 10-11). Como se afirma en la Declaración y Plataforma de Acción de Beijing: 
La función que cumplen las mujeres de dar y sustentar la vida les ha proporcionado aptitudes e ideas esenciales para unas relaciones humanas pacíficas y para el desarrollo social. Las mujeres se adhieren con menos facilidad que los hombres al mito de la eficacia de la violencia y pueden portar una amplitud, una calidad y un equilibrio de visión nuevos con miras al esfuerzo común que supone pasar de una cultura de guerra a una cultura de paz. (Organización de las Naciones Unidas, 1995b)

Otro ejemplo de las posiciones logradas y aportes de las mujeres en la obtención de la paz y el posconflicto es la teniente Zuly Vannesa Lugo, quien tuvo a cargo en el 2020 la misión de ser observadora militar para la ONU y verificar el cumplimiento del alto al fuego pactado entre Sahara Occidental y Marruecos, en medio del proceso independentista del primero. Así mismo, ella es la representación del Ejército Nacional colombiano en África, lo que significó, en sus palabras, el honor de ser la primera mujer de las FF. MM. en lograr un encargo de esa magnitud (Méndez, 2020).

Con evidencias como estas queda demostrado el valor de los aportes de las mujeres a las fuerzas armadas, labor exaltada por sus superiores, como el general Eduardo Enrique Zapateiro, quien afirmó que la teniente Lugo "es la primera mujer militar en realizar esta misión, lo que demuestra su profesionalismo, excelente preparación y condiciones militares que la hicieron sobresalir entre los demás miembros de la Fuerza" (Méndez, 2020).

\section{La mujer militar como agente articulador entre la soberanía y la paz en el Estado colombiano}

Las FF. MM., como parte de la estructura constitucional colombiana, tienen la misión especial de ser instrumento para el cumplimiento de los fines del Estado, así como de mantener el orden constitucional, garantizar y proteger los derechos y libertades de todos los habitantes en el territorio, y la defensa de la soberanía nacional. Por consiguiente, estas tendrán como una de sus funciones principales la consecución de la integridad e independencia del territorio nacional, tal y como lo establece el artículo 217 de la carta constitucional. Al tomar en cuenta que esta soberanía reside en el pueblo, las instituciones militares deben generar políticas de defensa y protección de los DD. HH. y del DIH de los ciudadanos. Es justamente en estas actividades que las mujeres militares cumplen una función fundamental, porque, desde su perspectiva, ellas toman medidas de defensa diferentes a los enfrentamientos bélicos y ayudan a la materialización de la cooperación con organizaciones humanitarias y civiles en la tarea de protección y de seguridad para lograr 
la paz. Todo ello tiene lugar gracias a la cooperación cívico-militar para favorecer el apoyo humanitario necesario para la población civil y el desarrollo de actividades orientadas a minimizar la baja de civiles en operaciones militares.

Es por esto que, para lograr una justicia de género dentro de las FF. MM. en la que se superen las desigualdades que afectan a la mujer, se debe brindar mayor participación para ellas en la toma de decisiones, en situaciones en las que sea necesario repeler ataques contra la sociedad civil y en las negociaciones para la obtención de la paz. De esta manera, ellas pasarán de ser una minoría subvalorada y relegada a los roles de género tradicionales a ser parte de los mandos superiores que toman las decisiones importantes y relevantes para la sociedad.

La Resolución 1325 de 2000 del Consejo de Seguridad de la ONU logra centralizar mecanismos para lograr un hito en los derechos humanos de las mujeres, la paz y la seguridad, promoviendo la participación de ellas, dada su mayor facilidad para tocar temas que se relacionan con la familia, la educación, la salud y la inequidad. Estas aptitudes representan mejor los intereses de la sociedad y los de su propio género, lo cual abre un camino más expedito para la construcción de una sociedad pacífica y justa, en la que hombres y mujeres trabajen de forma conjunta por el sueño de la paz.

\section{Conclusiones}

Desde 1976, con el Decreto 2129 que permitió el ingreso de mujeres al Ejército Nacional en la categoría de oficiales del cuerpo administrativo, se ha demostrado cómo el liderazgo de las mujeres rompió con la organización clásica del establecimiento de funciones dentro de las instituciones militares en Colombia. Dicho rompimiento ha sido del todo positivo, porque posibilitó la implementación de comunicaciones que favorecieron un entorno con mayor estabilidad emocional y se generó un ambiente más sano para la toma de decisiones y el establecimiento de estrategias colaborativas. Además, se hizo posible la participación de las mujeres en misiones de paz internacionales (negociaciones y procesos de desarme), lo que aumentó la eficacia de estas misiones, con criterios de veeduría, denuncias y priorización del goce mínimo de DD. HH.

Las mujeres, a través de sus esfuerzos por la igualdad y la equidad dentro de las instituciones castrenses, han logrado escalar a cargos de dirección y decisión dentro de estas. Esto ha hecho que se tome en cuenta su forma de pensar y su criterio como aporte a la solución de conflictos y la obtención de la paz. Todo ello ha sido altamente significativo para lograr la garantía de los derechos fundamentales y 
aspirar a una paz duradera, debido a que muchas veces sus aportes vienen desde sus vivencias y funciones diversas dentro de la sociedad. Esto último les da una visión que facilita el compromiso de una responsabilidad social que es fundamental para el logro de la convivencia pacífica y el respeto por los derechos humanos, objetivos de la humanización del conflicto.

A lo largo del tiempo siempre se ha hablado de la vulnerabilidad de las mujeres en el conflicto armado y de las consecuencias y secuelas que este deja para ellas en todos los aspectos, pero no es menos importante el papel que ellas han desarrollado en el posconflicto. Más allá de ser víctimas por recibir los daños causados por las partes del conflicto, se destacan como tejedoras de paz, como lideresas y servidoras públicas, labores que desarrollan día a día las mujeres militares, ya sea como combatientes o como profesionales que prestan sus servicios a las víctimas de estos conflictos. Sus aportes son de capital importancia frente a las consecuencias de la guerra que afectan a la sociedad civil, marco en el que su voz se escucha fuerte y sus aportes son parte de la solución.

\section{Referencias}

Barros, M. A., \& Rojas, N. (2015). La mujer en el conflicto colombiano: de víctima a empoderada. El libre pensador, 24. https://librepensador.uexternado.edu.co/la-mujer-en-el-conflicto-colombiano-de-victima-a-empoderada/

Beauvoir, S. (1949). El segundo sexo. Editorial Siglo Veinte.

Bobea, L. (2008). Mujeres en uniforme: la feminización de las fuerzas armadas. Un estudio del caso dominicano. Nueva Sociedad, 213, 64-79

Bordieu, P. (2000). La dominación masculina. Editorial Anagrama

Calderón, M. I., Patarroyo, D. B., \& Ramírez, L. M. (2017). Mujer, paz y posconflicto [Ponencia]. 9.o Congreso Latinoamericano de Ciencia Política, organizado por La Asociación Latinoamericana de Ciencia Política (ALACIP), Montevideo. https://bit.ly/36Y6Z5c

Calderón, Y. (2019, septiembre 26). Jéssica, la santandereana que lidera la misión de desminado humanitario en Colombia. Vanguardia. https:/www.vanguardia.com/area-metropolitana/ bucaramanga/jessica-la-santandereana-que-lidera-la-mision-de-desminado-humanitario-en-colombia-HD1476108

Camacho, C. M. (2014). Ejército, feminidades y género performativos: las experiencias de ser mujer y militar en la Escuela General de Cadetes "General José María Córdova" [Tesis de maestría]. Pontificia Universidad Javeriana.

Cely Calderón, M. P. (2019). Configuración de los roles de género en mujeres pertenecientes al Ejército Nacional de Colombia y sus implicaciones en el sistema familiar [Trabajo de grado]. Repositorio de la Universidad Externado de Colombia. https://bdigital.uexternado.edu.co/ bitstream/001/2336/1/DEA-spa-2019-Configuracion_de_los_roles_de_genero_en_mujeres_ pertenecientes_al_Ejercito_Nacional_de_Colombia 
Comando General de las Fuerzas Militares de Colombia. (2017). Mujeres militares: historias de grandeza al servicio de la paz. Acento.

Congreso de la República. (1976, octubre 7). Decreto 2129 de 1976.

Congreso de la República. (2000, mayo 31). Ley 581 de 2000.

Congreso de la República. (2016, febrero 26). Disposición 004 de 2016.

Constitución Política de Colombia. (1991). Legis Editores (42 Ed., 2019).

De la Hoz Flórez, M. P. (2018). La mujer en las Fuerzas Armadas de Colombia y España: agente de reconstrucción social durante el posconflicto. Universidad Católica de Colombia.

Donadio, M., Klepak, H., Kussrow, S., Pabón Ayala, N., \& Rial, J. (s. f.). Misiones militares y posconflito: una mirada regional sobre Colombia. Resdal (Red de Seguridad y Defensa de América Latina. https://www.resdal.org/assets/diagnostico_colombia_resdal-esp-2018.pdf

Gallardo, P. (2012). La mujer militar en las Fuerzas Armadas. Cuadernos de Estrategia, 157, 139-174.

Huertas Díaz, O., Ruiz Herrera, A. L., \& Botía Hernández, N. J. (2017). De mujer combatiente a mujer constructora de paz. Inclusión de la voz femenina en el escenario del posacuerdo. Ratio Juris, 12(25), 43-68.

Hussain-Talero, S. (2018). La mujer uniformada en la fuerza pública colombiana: un estudio sobre su liderazgo. En A. E. Fernández Osorio, \& E. J. Latorre Rojas (Eds.), La construcción del rol de la mujer militar (pp. 93-116). Escuela Militar de Cadetes "General José María Córdova”.

Instituto de Estudios sobre Conflictos y Acción Humanitaria. (2010). La participación militar en la acción humanitaria. Reflexiones en torno al caso colombiano.

Londoño, J. D. (2017). La mujer en la construcción de paz: la visión de la General Galvis. En A. M. Caicedo (Dir.), Mujeres militares: historias de la grandeza al servicio de la paz. ONU Mujeres; Comando General de las Fuerzas Militares.

Méndez, A. L. (31 de enero de 2020). ¿Quién es la primera militar colombiana en ir a África con la ONU? El Tiempo. https://www.eltiempo.com/justicia/servicios/la-teniente-colombiana-primera-mujer-militar-de-observadores-de-onu- 457268

Millán, J. (2017, febrero 5). Lo que nunca imaginé: Juanita Millán. El Espectador. https://www. elespectador.com/colombia2020/pais/lo-que-nunca-imagine-juanita-millan-articulo-855152/

Naciones Unidas. (2014). Cuestiones de género: mujeres, paz y seguridad en el Departamento de Asuntos Políticos. https://www.un.org/undpa/es/women-peace-security

Organización de las Naciones Unidas - Consejo de Seguridad. (2000, octubre 31). Resolución 1325 (2000).

Organización de las Naciones Unidas. (1994, junio 9). Convención Interamericana para Prevenir, Sancionar y Erradicar la Violencia contra la Mujer "Convención de Belém do Pará". Belém do Pará.

Organización de las Naciones Unidas. (1995a, octubre 17). Cuarta Conferencia Mundial sobre la Mujer. Beijing, China.

Organización de las Naciones Unidas. (1995b). Declaración y Plataforma de Acción de Beijing. https://beijing20.unwomen.org/es/about

Organización de las Naciones Unidas. (2000, julio 12). Carta dirigida al secretario general por el representante permanente de Namibia ante las Naciones Unidas. https://undocs.org/pdf?symbol=es/S/2000/693 
Ramírez, S. (2016, agosto). Poniéndole género a la paz. Nueva Sociedad (nuso.org). https://nuso.org/ articulo/poniendole-genero-la-paz/

Sentencia C-225/95. Corte Constitucional. Magistrado Ponente: Alejando Martínez Caballero, 18 de mayo de 1995.

Sentencia C-408/96, Corte Constitucional. Magistrado Ponente: Alejandro Martínez Caballero, 4 de septiembre de 1996.

Sentencia C-438/13, Corte Constitucional. Magistrado Ponente: Alberto Ríos Rojas, 10 de Julio de 2013.

Sentencia C-659/16, Corte Constitucional. Magistrado Ponente: Aquiles Arrieta Gómez, 28 de noviembre de 2016.

Valencia Tovar, G. A. (1994). Fuerzas Armadas y derechos humanos. En R. Cerdas Cruz, \& R. Nieto Loaiza (Comps.), Estudios Básicos de Derechos Humanos I. Instituto Interamericano de Derechos Humanos.

Vargas, J., \& Díaz Pérez, A. (2018). Enfoque de género en el acuerdo de paz entre el Gobierno Colombiano y las FARC-EP: transiciones necesarias para su implementación. Revista Iberoamericana de Filosofía, Politica y Humanidades, 20(39), 389-414. https://doi.org/10.12795/ araucaria.2018.i39.19 